\title{
Who Experience Higher Level of Anxiety During COVID-19 Outbreak?
}

Marzieh Haghayeghi ${ }^{1}$, Ali Moghadam Zadeh ${ }^{2 *}$, Amin Mousavi ${ }^{3}$, Seyedeh Hoda Naji ${ }^{4}$

${ }^{1}$ Department of Curriculum Development \& Instruction Methods, Faculty of Psychology and Education, University of Tehran, Iran.

${ }^{2}$ Assistant Professor, Department of Curriculum Development \& Instruction Methods, Faculty of Psychology and Education, University of Tehran, Iran

${ }^{3}$ Department of Educational Psychology and Special Education, College of Education, University of Saskatchewan, Canada.

${ }^{4}$ Department of Curriculum Development \& Instruction Methods, Faculty of Psychology and Education, University of Tehran, Iran.

*Correspondence: Ali Moghadam Zadeh, Assistant Professor, Department of Psychology, Faculty of Psychology and Education, University of Tehran, Tehran, Iran. Email: amoghadamzadeh@ut.ac.ir

Received Date: July 31, 2020; Accepted Date: September 20, 2020; Published Date; November 30, 2020

Citation: Marzieh Haghayeghi,Ali Moghadam Zadeh, Amin Mousavi, Seyedeh Hoda Naji, Who Experience Higher Level of Anxiety During COVID-19 Outbreak?; J. Psychology and Mental Health Care. 5(1). Doi: 10.31579/2637-8892/093.

Copyright: ( 2020 Raquel Rodríguez-Alcántara, This is an open-access article distributed under the terms of the Creative Commons Attribution License, which permits unrestricted use, distribution, and reproduction in any medium provided the original author and source are credited.

\begin{abstract}
This study was conducted with the assumption that there would be a significant and negative relationship between Coronavirus Anxiety (CVA) and Psychological Well-Being (PWB). However, the findings did not meet our assumption because one of the components of PWB named "purposefulness-in-life" had a strong and positive correlation with CVA, meaning that those participants with a strong goal orientation would experience higher level of CVA. To our knowledge, this finding could be considerable in the new situation of COVID-19 pandemic. In the following analysis, the moderation effects of age and gender on the relationship between CVA and purposefulness-in-life were found significant.
\end{abstract}

Keywords: coronavirus anxiety; psychological well-being; purposefulness-in-life; covid-19 outbreak

Abbreviations: Corona-virus Anxiety (CVA), Psychological Well-being (PWB), World Health Organization (WHO)

\section{Introduction}

On 11 March 2020, WHO declared COVID-19 disease as a pandemic that affected people all over the world (World Health Organization, 2020). As a result of this global health crisis, stringent public health measures have been implemented to prevent COVID-19 from spreading(Adhikari et al., 2020). One usual policy against such pandemics is a quarantine which is often an unpleasant experience for those undergoing it (Moccia et al., 2020). Given the evidence from similar epidemics and pandemics, in such cases, quarantine can cause feelings of loneliness and anger among people losing face-to-face connections and traditional social interactions (Xiang et al., 2020; Zandifar \& Badrfam, 2020; Zhang, Wu, Zhao, \& Zhang, 2020). All these containment measures have a strong impact on the population's daily life and may negatively affect their psychological wellbeing (Rajkumar, 2020).

The coronavirus outbreak and the policies used for managing it, such as quarantine, have negative socioeconomic effects on mental health, not only due to the loss of freedom and separation from each other as well as uncertainty over disease status (Brooks et al., 2020), but also due to an increase in unemployment, financial insecurity and poverty (Holmes et al., 2020). Therefore, this situation may have the potential to result in a parallel global pandemic of mental health morbidity throughout the world. According to WHO's web-site, the main psychological impact of COVID-19 on public mental health to date is elevated rates of stress or anxiety (World-Health-Organization, 2020).
Regarding the potentially negative effects of anxiety caused by COVID-19 outbreaks on public mental health, this web-based survey with the purpose of investigating the relationship between Corona-virus Anxiety (CVA) and Psychological Well-being (PWB) was conducted in Iran as a country that reported high rate of mortality by COVID-19.

\section{Methods and Procedures}

The survey consisted of sociodemographic questions and two wellestablished scales: (1) The Coronavirus Anxiety (CVA) Scale with 9 items that measures the anxiety caused by the prevalence of coronavirus. Each item response ranged from 1 to 4 (i,e, 1=Never and $4=$ always). Higher scores indicate higher levels of CVA. The Guttman's $\lambda$ value and Cronbach's alpha coefficient for this questionnaire were reported as $\lambda=$ 0.922 and $\alpha=0.919$ respectively (Alipour, Ghadami, Alipour, \& Abdollahzade, 2020). (2) The short Version of the Ryff Scales of Psychological Well-Being (PWB-18-items) which was designed in a 6point Likert scale (from 1=completely disagree to $6=$ completely disagree), and was use for measuring the six sub-scales of PWB including "self-acceptance", "environmental mastery", "positive relationships with others", "purposefulness-in-life", "personal growth" and "autonomy" (Ryff, 1989). Its Persian version was adapted for Iranian community with a reliability coefficient of $\alpha=0.83$ for the whole scale and $0.65<\alpha<0.75$ for its six sub-scales (Sefidi \& Farzad, 2012). In this study, the internal consistency using Cronbach's alpha was 0.89 and 0.76 for the two questionnaires, respectively. The Cronbach's alphas for the six sub-scales of PWB-18 were between 0.61 and 0.73 . 
The URL of the questionnaires, which had been approved by the Ethics Committee of Ministry of Science, Research and Technology of Iran (number: IR.SSRC.REC.1399.026), was sent to those who were easily available via Facebook and some networking applications such as WhatsApp ${ }^{\mathrm{TM}}$, Telegram ${ }^{\mathrm{TM}}$ and Instagram ${ }^{\mathrm{TM}}$ between April $25^{\text {th }}$ and May $15^{\text {th }}$ of 2020 . The inclusion criteria was the age participants between 18 and 60. Exclusion criteria were: a recent hospitalization or a history of mental disorder. A total of 974 participants answered our online questionnaires; 75 respondents $(7.7 \%)$ were under 18 or more than 60 years old, and since they did not meet our inclusion criteria, they were excluded. Also 31 questionnaires (3.2\%) were put aside due to incomplete responses. Therefore, 868 questionnaires were used for the final analysis.

\section{Data Analysis}

SPSS $_{26}$ was utilized for analyzing descriptive statistics (frequency, mean and standard deviation) and inferential statistics (independent t-test, Pearson's correlation and regression). And SPSSPROCESS, Macro Hayes's version 3.5 was used for moderation analysis.

\section{Findings}

The sample consisted of $65 \%$ females $(n=564)$ and $36 \%$ single persons $(n=313)$. The mean age of the sample was $34.97(\mathrm{SD}=9.69)$.

The mean score of CVA-9 was $18.72(\mathrm{SD}=5.73)$ and the results showed that females $(M=19.02, S D=5.8)$ experience higher level of CVA than males $(\mathrm{M}=18.16, \mathrm{SD}=5.6)$, and this difference was statistically significant $(\mathrm{t}=-2.12$, P-value $<0.05)$. The mean score of PWB-18 was 83.54 $(\mathrm{SD}=10.71)$, and the difference between males $(\mathrm{M}=83.12, \mathrm{SD}=10.6)$ and females $(\mathrm{M}=84.31, \mathrm{SD}=10.78)$ was not statistically significant $(\mathrm{t}=1.55, \mathrm{P}$ value $>0.05$ ).
The total score of PWB was negatively correlated with CVA $(r=-0.24$, Pvalue $<0.05$ ). The correlations between CVA and the sub-scales of PWB including "self-acceptance", "environmental mastery", "positive relationships with others", "purposefulness-in-life", "personal growth" and "autonomy" were $-0.21,-0.23,-0.15,0.78,-0.13$ and -0.12 respectively (all of the correlations coefficients were statistically significant at $\mathrm{P}$-value $<0.01)$. What is considerable about these correlations is that all of the PWB's sub-scales correlated negatively with CVA, except "purposefulness-in-life" which had a strong and positive correlation with CVA (i.e, 0.78).

Regression analysis was used to examine how much of CVA's variance could be explained by the subscales of $\mathrm{PWB}$, the Considering the variance of CVA explained by sub-scales of PWB $\left(\mathrm{R}^{2}=0.61, \mathrm{~F}=221.75\right.$, $\mathrm{P}$-value < $0.000)$, the "purposefulness-in-life" positively predicted the CVA $(\beta=$ $0.77, \mathrm{t}=34.53$, P-value $<0.000$ ), while other sub-scales of PWB could not significantly predict CVA $(-0.06<\beta<0.3,-1.97<\mathrm{t}<1.28,0.051<\mathrm{P}$-value $<0.7$ ). Indeed, 60 percentage of the variance of CVA is explained by purposefulness-in-life $\left(\mathrm{R}=0.78, \mathrm{R}^{2}=0.60, \mathrm{~F}=1309.05, \mathrm{P}\right.$-value $<0.000$, $\beta=0.78, t=36.18)$.

With respect to the strong relationship found between CVA and purposefulness-in-life, following statistical analysis concentrated on moderation effects of "age" and "gender" on this relationship. Findings revealed that age $(b=0.0009, \mathrm{se}=0.0004, \mathrm{P}$-value $=0.030)$ and gender $(b=$ 0.017 , $\mathrm{se}=0.0082, \mathrm{P}$-value $=0.036$ ) were the significant moderators of the effect of purposefulness-in-life on CVA. The conditional effects of age $(b=-0.0048$, s.e. $=0.002$, p-value $=0.014)$ and gender $(b=-0.12$, s.e. $=0.041$, $\mathrm{p}$-value $=0.007$ ) on CVA were negative and significant, conditional on purposefulness-in-life $=0$. The interactions of CVA and purposefulnessin-life in term of age and gender are visualized in Fig.1 and Fig.2 respectively.

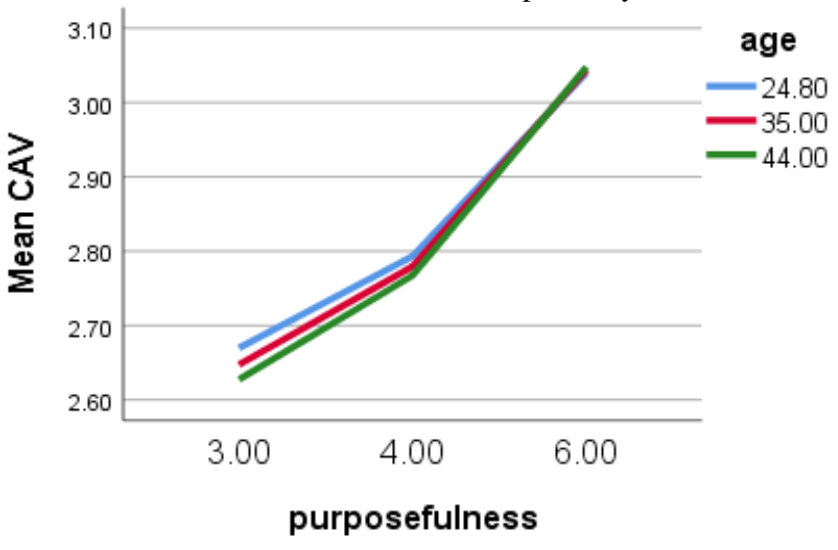

Fig. 1. The relationship between purposefulness-in-life $(\mathrm{X})$ and CAV (Y) at different levels of the moderator $(\mathrm{W}=\mathrm{age})$.

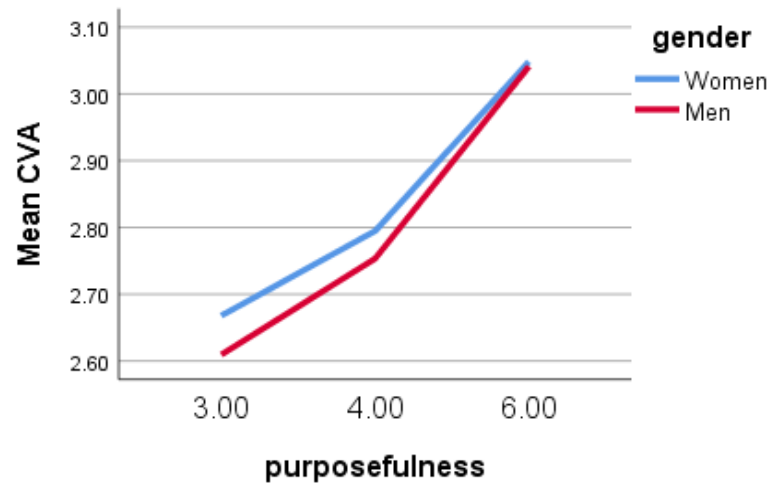

Fig. 2. The relationship between purposefulness-in-life (X) and CAV (Y) at different levels of the moderator (W= gender). 
In respect of Fig.1, the relationship between purposefulness-in-life and CVA is more strongly positive at $-3 \mathrm{SD}$ on the centered age variable and less strongly negative at the - 1SD on that variable. Regarding Fig.2, the effect of purposefulness-in-life on CVA is more strongly positive among men.

\section{Discussion}

We conducted this study with the assumption that individuals having better Psychological Well-Being (PWB) experience less anxiety (Nakazawa et al., 2018). This study was carried out during COVID-19 outbreaks and the findings did not support our hypothesis.

However, the most important component of PWB called purposefulnessin-life (Ryff, 2014) has been proven to result in less anxiety(Bonebright, Clay, \& Ankenmann, 2000; Ryff, 1989). In this study, it had a strong positive relationship with CVA, which means that individuals having a strong sense of purpose in their lives feel higher level of anxiety during coronavirus pandemic.

There are some probable reasons explaining why individuals who set goals and define purpose in their lives experience higher level of anxiety during coronavirus pandemic in comparison to those who do not commit themselves to a definite purpose. On the one hand, the key parts of purposefulness-in-life including social connections, engagement and sense of community (Johnson, Park, \& Chaudhuri, 2020) are under threat of isolation due to quarantine. And people's lifestyles have completely been changed because of COVID-19 outbreaks. On the other hand, COVID-19 disease expose people to a situation that is not predictable. Therefore, people with a strong goal orientation may struggle not only with achieving their pre-definite goals, but also with making a new plan for their life in this unexpected situation.

After reviewing the researches and interactions that have been raised around PWB, Ryff (2014) stated that the findings are complex, depending on the age and gender (Ryff, 2014). Anyway, with respect to Iranian culture in which men are supposed to be more responsible for earning a living than women, as well as with respect to some socioeconomical effects of COVID-19 outbreaks; gender and age played moderating roles in regard to the effect of purposefulness-in-life on CVA. Since this study was conducted in the Iranian population during coronavirus epidemic, caution should be exercised in generalizing the findings to other communities. And causal relationships of variables are necessary to be investigated by experimental designs.

\section{Author Agreement Statement:}

We the undersigned declare that this manuscript is original, has not been published before and is not currently being considered for publication elsewhere. We confirm that the manuscript has been read and approved by all named authors and that there are no other persons who satisfied the criteria for authorship but are not listed. We further confirm that the order of authors listed in the manuscript has been approved by all of us. We understand that the Corresponding Author is the sole contact for the Editorial process. He is responsible for communicating with the other authors about progress, submissions of revisions and final approval of proofs.

Signed by all authors,

\section{Ethical Statement:}

The study was approved by the Ethics Committee of Ministry of Science, Research and Technology of Iran((number: IR.SSRC.REC.1399.026)). Participants were mentioned to be able not to continue the survey at any time without providing any justification. In addition, we gave the scores and status reports to those participants who asked for them. The data was collected anonymously and confidentiality was preserved in keeping with privacy policy.

\section{Signed by all authors}

\section{Conflicts of Interest Statement:}

The authors whose names are listed immediately below certify that they have NO affiliations with or involvement in any organization or entity with any financial interest (such as honoraria; educational grants; participation in speakers' bureaus; membership, employment, consultancies, stock ownership, or other equity interest; and expert testimony or patent-licensing arrangements), or non-financial interest (such as personal or professional relationships, affiliations, knowledge or beliefs) in the subject matter or materials discussed in this manuscript.

Marzieh Haghayeghi: PhD student,Department of Curriculum Development \& Instruction Methods, Faculty of Psychology and Education, University of Tehran, Iran, email: m.haghayegi@ut.ac.ir

Ali Moghadam Zadeh* : Assistant Professor, Department of Curriculum Development \& Instruction Methods, Faculty of Psychology and Education, University of Tehran, Iran e-mail: amoghadamzadeh@ut.ac.ir

Amin Mousavi : Department of Educational Psychology and Special Education, College of Education, University of Saskatchewan, Canada, amin.mousavi@usask.ca

Seyedeh Hoda Naji: PhD student,Department of Curriculum Development \& Instruction Methods, Faculty of Psychology and Education, University of Tehran, Iran, email: m.haghayegi@ut.ac.ir

\section{References}

1. Adhikari, S. P., Meng, S., Wu, Y.-J., Mao, Y.-P., et al (2020). Epidemiology, causes, clinical manifestation and diagnosis, prevention and control of coronavirus disease (COVID-19) during the early outbreak period: a scoping review. Infectious diseases of poverty, 9(1), 1-12.

2. Alipour, A., Ghadami, A., Alipour, Z., and Abdollahzade, H. (2020). Preliminary Validation of the Corona Disease Anxiety Scale (CDAS) in the Iranian Sampl. Quarterly Journal of Health Psychology, 8, 163-175.

3. Bonebright, C. A., Clay, D. L., and Ankenmann, R. D. (2000). The relationship of workaholism with work-life conflict, life satisfaction, and purpose in life. Journal of counseling psychology, 47(4), 469.

4. Brooks, S. K., Webster, R. K., Smith, L. E., Woodland, L., Wessely, S., et al (2020). The psychological impact of quarantine and how to reduce it: rapid review of the evidence. The Lancet.

5. Holmes, E. A., O'Connor, R. C., Perry, V. H., Tracey, I., Wessely, S., et al (2020). Multidisciplinary research priorities for the COVID-19 pandemic: a call for action for mental health science. The Lancet Psychiatry.

6. Johnson, K. R., Park, S., and Chaudhuri, S. (2020). Mindfulness training in the workplace: exploring its scope and outcomes. European Journal of Training and Development.

7. Moccia, L., Janiri, D., Pepe, M., Dattoli, L., Molinaro, M., et al (2020). Affective temperament, attachment style, and the psychological impact of the COVID-19 outbreak: an early report on the Italian general population. Brain, Behavior, and Immunity.

8. Nakazawa, K., Noda, T., Ichikura, K., Okamoto, T., Takahashi, Y., Y, et al (2018). Resilience and depression/anxiety symptoms in multiple sclerosis and neuromyelitis optica spectrum disorder. Multiple sclerosis and related disorders, 25, 309-315.

9. Rajkumar, R. P. (2020). COVID-19 and mental health: A review of the existing literature. Asian journal of psychiatry, 102066.

10. Ryff, C. D. (1989). Happiness is everything, or is it? Explorations on the meaning of psychological well-being. Journal of personality and social psychology, 57(6), 1069. 
11. Ryff, C. D. (2014). Psychological well-being revisited: Advances in the science and practice of eudaimonia. Psychotherapy and psychosomatics, 83(1), 10-28.

12. Sefidi, F., and Farzad, V. (2012). Validated measure of Ryff psychological well-being among students of Qazvin University of Medical Sciences (2009). The Journal of Qazvin University of Medical Sciences, 16(1), 65-71.

13. World-Health-Organization. (2020). Emergencies preparedness, response.

14. Xiang, Y.-T., Yang, Y., Li, W., Zhang, L., Zhang, Q., et al (2020). Timely mental health care for the 2019 novel coronavirus outbreak is urgently needed. The Lancet Psychiatry, 7(3), 228229.

15. Zandifar, A., and Badrfam, R. (2020). Iranian mental health during the COVID-19 epidemic. Asian journal of psychiatry, 51, 101990.

16. Zhang, J., Wu, W., Zhao, X., and Zhang, W. (2020). Recommended psychological crisis intervention response to the 2019 novel coronavirus pneumonia outbreak in China: a model of West China Hospital. Precision Clinical Medicine, 3(1), 3-8. 